\title{
Decreased Renal Function Is Associated with Elevated CHA2DS2VASC and R2CHADS2 Scores in Non-Valvular Atrial Fibrillation Patients Presenting with Stroke
}

Mohinder R. Vindhyal ${ }^{1}$, Shravani R. Vindhyal ${ }^{2}$, Travis Haneke ${ }^{2}$, Paul M. Ndunda ${ }^{2}$, Freidy Eid $^{3}$, K. James Kallail ${ }^{2}$

1. Internal Medicine, University of Kansas Medical School Wichita, Andover, USA 2. Internal Medicine, University of Kansas School of Medicine - Wichita 3. Cardiology, Robert J Dole Va Hospital

$\square$ Corresponding author: Mohinder R. Vindhyal, mvindhyal@hotmail.com

Disclosures can be found in Additional Information at the end of the article

\section{Abstract}

\section{Introduction}

Atrial fibrillation (AF), the most common cardiac arrhythmia, affects approximately 2.3 million patients in the United States, costing around $\$ 26$ billion. Atrial fibrillation is associated with a two- to seven-fold increased risk of stroke, one of the most serious complications. Chronic kidney disease affects approximately $13 \%$ of the US population and has been associated with higher rates of AF than the general population. In patients with chronic kidney disease (CKD), the risk of stroke increases as the glomerular filtration rate (GFR) decreases, especially in CKD stages three and four.

Several risks stratification scores such as CHADS2 (congestive heart failure, hypertension, age, diabetes mellitus, stroke), CHA2DS2VASc (congestive heart failure, hypertension, age, diabetes mellitus, stroke, vascular disease, age, sex), and R2CHADS2 (renal failure, congestive heart failure, age, diabetes, stroke) scores are used for stroke risk assessment in patients with nonvalvular atrial fibrillation (NVAF). This study investigates the association between renal functions and risk stratification scoring systems in patients with non-valvular AF presenting with stroke.

\section{Methods}

Using the convenience sampling method, 171 subjects were selected from the eligible population ( $\mathrm{n}=386)$. A Pearson product-moment correlation coefficient was calculated to

Received 11/28/2017 Review began 12/04/2017 Review ended 12/05/2017 Published 12/11/2017

\section{(c) Copyright 2017}

Vindhyal et al. This is an open access article distributed under the terms of the Creative Commons Attribution License CC-BY 3.0., which permits unrestricted use, distribution, and reproduction in any medium, provided the original author and source are credited. determine the association between the GFR and each of the CHA2DS2VASc and R2CHADS2 scores. In addition, a Pearson product-moment correlation coefficient was calculated to determine the association between the CHA2DS2VASc and R2CHADS2 scores.

\section{Results}

The selected population represented $44.3 \%$ of the eligible subjects. Of these, $88 \%$ were Caucasian, $60 \%$ were female, and the mean age was 78 years. The mean CHA2DS2VASc score was six (range 2-9). The mean eGFR was 69.77 (range 6-108). Both the mode and the median CHA2DS2VASc score was four (range 2-8). A weak, but significant, negative correlation was found between renal function (eGFR) and the CHA2DS2VASc score $(r=-0.263 ; p=0.0005)$. There was a stronger negative correlation between the eGFR and R2CHADS2 score $(r=-0.70$; $p$

\section{How to cite this article}

Vindhyal M R, Vindhyal S R, Haneke T, et al. (December 11, 2017) Decreased Renal Function Is Associated with Elevated CHA2DS2VASC and R2CHADS2 Scores in Non-Valvular Atrial Fibrillation Patients Presenting with Stroke. Cureus 9(12): e1935. DOI 10.7759/cureus.1935 
$<0.00001)$. The CHA2DS2VASc and R2CHADS2 scoring schemes were significantly and positively correlated $(\mathrm{r}=0.627 ; \mathrm{p}<0.00001)$.

\section{Discussion}

In NVAF patients presenting with stroke, renal failure is associated with higher CHA2DS2VASc and R2CHADS2 scores. One must consider renal failure (end-stage or non-end stage renal failure) as an additional potential risk factor for stroke when recommending anticoagulation in non-valvular atrial fibrillation.

Categories: Cardiology, Internal Medicine, Nephrology

Keywords: non-valvular atrial fibrillation, renal failure, stroke

\section{Introduction}

Atrial fibrillation (AF) is the most common cardiac arrhythmia [1] affecting approximately 2.3 million Americans. The number is expected to increase to 5.6 million by 2050 [2]. Every year, AF costs around $\$ 26$ billion, causing a substantial burden to the healthcare system [3]. Atrial fibrillation is associated with a two- to seven-fold increased risk of stroke, one of the most serious complications [4]. Many risk factors contributing to AF have been identified; among them, the non-modifiable factors are age and gender. Other factors include hypertension, valvular heart disease, congenital heart disease, heart failure, coronary artery disease, obesity, diabetes mellitus, smoking, alcohol consumption, thyroid disease, and chronic kidney disease [5]. Of the many risk factors for AF, chronic kidney disease (CKD) is a potentially modifiable risk factor. Chronic kidney disease affects approximately $13 \%$ of the US population [6] and has been associated with higher rates of AF than the general population [7]. In patients with CKD, the risk of stroke increases as the glomerular filtration rate (GFR) decreases, especially in CKD stages three and four [8].

Both AF and CKD increase the risk of thromboembolism. In the US, approximately 800,000 new strokes are reported yearly [9], which can have severe and devastating effects. Because of the potential for severe sequela from stroke, several risk stratification scores have been developed to aid in identifying patients requiring oral anticoagulation $(\mathrm{OAC})$ to prevent future thromboembolic events [10]. The most popular scores are the CHADS2 (congestive heart failure, hypertension, age, diabetes mellitus, stroke), CHA2DS2VASc (congestive heart failure, hypertension, age, diabetes mellitus, stroke, vascular disease, age, sex), and R2CHADS2 (renal failure, congestive heart failure, age, diabetes, stroke) scores. While each has a unique scoring system, they are alike in that they score one point for congestive heart failure, hypertension, and diabetes, and two points for previous episodes of stroke or thromboembolism. In the initial CHADS2 score, age greater than or equal to 75 years is given one point. However, in the updated CHA2DS2VASc score, one point is given for ages 65-74 years and two points for ages greater than or equal to 75 years. The latter scoring system also adds one point for vascular disease and the female sex. Neither of these risk stratification scores includes renal failure as a predictive prognostic factor, and both are endorsed by American College of Cardiology/American Heart Association (ACC/AHA). R2CHADS2 is another scoring system which includes two points for renal failure (stage 2 or greater) and stroke and one point for congestive heart failure, hypertension, and diabetes.

Patients with CKD and AF share many of the same risk factors for stroke; it is unclear if renal failure is itself an independent risk factor. Previous studies have attempted to evaluate the addition of renal failure as an independent variable to current risk stratification schemes [11]. However, these studies have suggested conflicting results as to whether or not renal failure serves as an independent risk factor in predicting stroke. 
This study aims to evaluate renal failure as a risk factor for thromboembolic events in patients previously diagnosed with nonvalvular atrial fibrillation (NVAF). Improvements in stroke risk stratification scores can further aid in properly determining which patients should receive anticoagulation. The information could be useful in reducing the number of adverse effects, such as hemorrhage or stroke, from incorrect use of therapies.

\section{Materials And Methods}

\section{Research study design}

This is a retrospective chart review.

\section{Participants}

Patients were selected from a Kansas hospital database from 2012 through 2014. To be included, patients older or of 18 years of age must have presented to the hospital with a stroke and a prior diagnosis of atrial fibrillation. There was no risk to patients.

\section{Instruments}

Patients meeting all inclusion criteria had the following parts of their charts reviewed: history and physical, lab data, admission echocardiogram, and discharge documents only from the current admission. The study variables included: medical record number, age, race, ejection fraction, creatinine, and history of hypertension, diabetes, vascular disease, congestive heart failure, and prior stroke. This study defined heart failure as either any diastolic heart failure or a systolic ejection fraction less than $40 \%$ as reported on an echocardiogram performed during the current hospital admission. Creatinine was collected to assess renal function, and the lowest creatinine value during the current hospitalization was selected for use as the best estimate of the patient's baseline value. Data on race was also collected to determine the estimated GFR using the CKD-EPI creatinine equation [12]. Calculation of the CHA2DS2VASc risk stratification score included assigning one point for the following: congestive heart failure, hypertension, age 65-74 years, diabetes mellitus, vascular disease (history of myocardial infarction, peripheral vascular disease, or aortic atherosclerosis), and female sex. Age greater than or equal to 75 years was assigned two points as was a prior history of stroke. Scoring for CHADS2 was one point for congestive heart failure, hypertension, age greater than or equal to 75 years, and diabetes mellitus, and two points were assigned for stroke. To calculate the R2CHADS2 score, the CHADS2 scoring system was used with an additional two points assigned for renal failure, which was defined as a calculated GFR less than 60 from the CDK-EPI equation.

\section{Procedures}

This study was approved by the institutional review boards at the University of Kansas School of Medicine-Wichita and Via Christi Hospitals of Wichita, KS. A convenience sample of 171 subjects from all eligible patients $(n=386)$ meeting the study criteria was assigned by the study director for review. The charts were reviewed by investigators, and the aforementioned data was collected and entered into a data sheet stored on password-secured computers. The data sheets were compiled into one data sheet for analysis. A convenience sample was used because as it was more a pilot study to obtain basic data and trends regarding the association. The demographics of the patient population can be seen in Table 1 . 


\section{Cureus}

\begin{tabular}{|c|c|c|c|}
\hline & Number of Subjects (\%) & Mean GFR (CKD - EPI) & Number of Subjects with Renal Failure (\%) \\
\hline ALL PATIENTS & $171(100)$ & 69.77 & $49(28.7)$ \\
\hline \multicolumn{4}{|l|}{ SEX } \\
\hline Male & 69 & 70.90 & $19(11.1)$ \\
\hline Female & 102 & 69.01 & $30(17.5)$ \\
\hline \multicolumn{4}{|l|}{ AGE } \\
\hline$<65$ & $23(13.5)$ & & \\
\hline $65-75$ & $37(21.6)$ & & \\
\hline$>75$ & $111(64.9)$ & & \\
\hline \multicolumn{4}{|l|}{ COMORBIDITIES } \\
\hline Heart failure & $54(31.6)$ & & \\
\hline Hypertension & $143(21.6)$ & & \\
\hline Diabetes & 57 (33.3) & & \\
\hline Vascular disease & $79(46.2)$ & & \\
\hline
\end{tabular}

\section{TABLE 1: Clinical characteristics of patients}

GFR: glomerular filtration rate

CKD - EPI: chronic kidney disease epidemiology collaboration

\section{Analysis}

A Pearson product-moment correlation coefficient was calculated to determine the association between the GFR and each of the CHA2DS2-VASc and R2CHADS2 scores. In addition, a Pearson product-moment correlation coefficient was calculated to determine the association between the CHA2DS2-VASc and R2CHADS2 scores. Summary statistics were calculated for each dependent variable.

\section{Results}

Of the total, 386 subjects met the eligibility criteria. A convenience sample of 171 subjects was selected, thus representing $44.3 \%$ of all eligible subjects. Among those sampled, $88 \%$ were Caucasian and $60 \%$ were female. The mean age was 78 years. The mean CHA2D2VASc score was six (range 2-9). The mean eGFR was 69.8 (range 6-108). Both the mode and the median R2CHA2D2VASc score was four (range 2-8). A weak, but significant, negative correlation was found between renal function (eGFR) and the CHA2DS2-VASc score (Figure 1; $r=-0.263 ; \mathrm{p}=$ 0.0005). There was a stronger negative correlation between the eGFR and R2CHADS2 scores (Figure 2; $\mathrm{r}=-0.70 ; \mathrm{p}<0.00001$ ). The CHA2DS2-VASc and R2CHADS2 scoring schemes were 


\section{Cureus}

significantly and positively correlated $(\mathrm{r}=0.627 ; \mathrm{p}<0.00001)$.

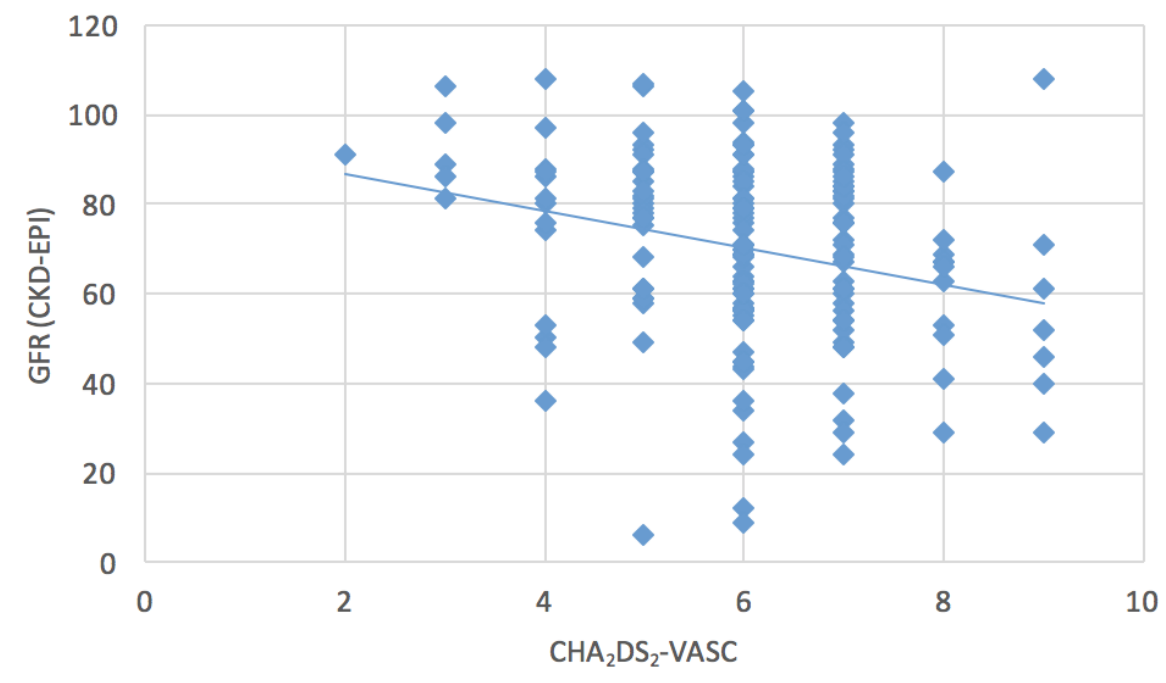

FIGURE 1: Correlation between CHA2DS2-VASC and GFR ( $r=$ $0.263 ; p=0.0005)$

GFR: glomerular filtration rate

CHA2DS2-VASC: congestive heart failure, hypertension, age, diabetes mellitus, stroke, vascular disease, age, sex

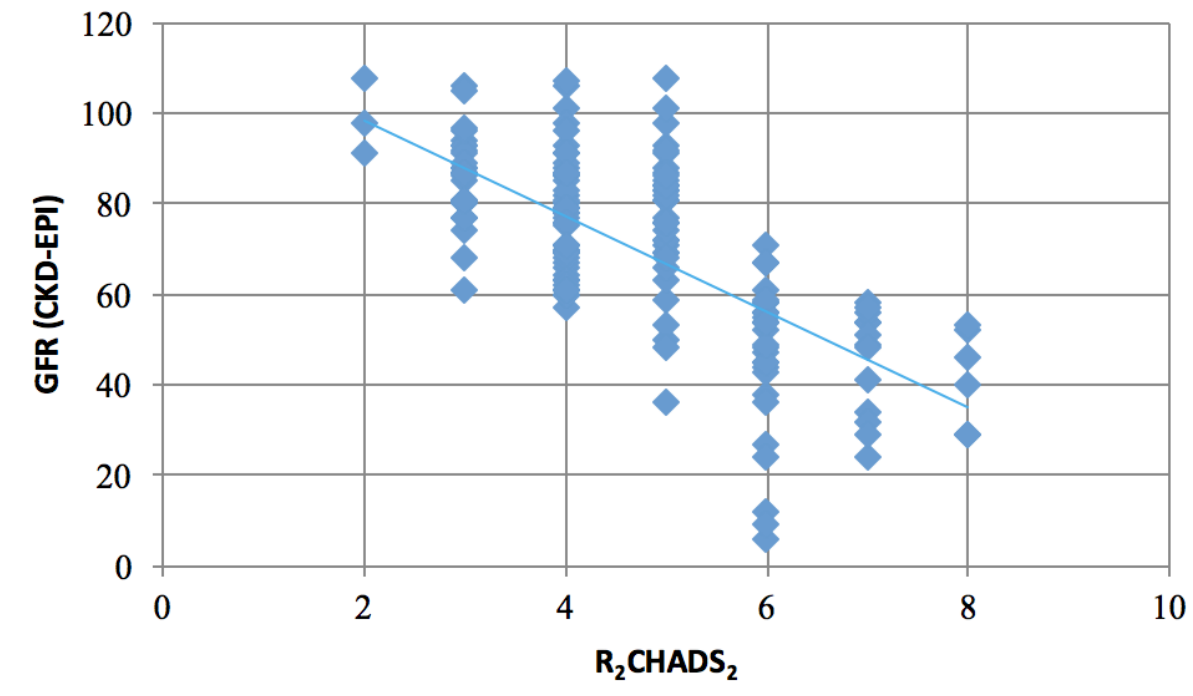

FIGURE 2: Correlation between R2CHADS2 and GFR ( $r=-0.70$; $p<0.00001)$ 


\section{Discussion}

This study highlights that in patients with NVAF, renal failure is associated with increased risk of stroke. At first glance, the negative correlation may be a bit confusing. As eGFR decreases, the CHA2DS2VASc score increases. Because a higher CHA2DS2VASc is associated with a higher risk of stroke, this means that as eGFR decreases, the yearly risk of stroke increases. The correlation between the eGFR and CHA2DS2VASc score also suggests the possibility that the risk of stroke progressively increases the further eGFR declines; however, the exact extent of this association is not well established. Alternatively, it is possible that no true relationship exists between these two variables but, rather, it is merely due to the sharing of the same risk factors. It is of note that this study does not compare renal failure with other risk factors in stroke risk stratification schemes in their ability to predict yearly stroke risk.

Another, yet stronger, negative correlation was found between eGFR and the R2CHADS2 score. Again, this suggests that renal failure, in this particular scoring system, is also associated with a higher risk of stroke. It is not surprising that this correlation exists and is stronger since the R2CHADS2 score includes renal failure as one of its components.

Additionally, there is a significant positive correlation between the CHA2DS2VASc and R2CHADS2 scores. This should not be a surprise as the two scoring systems share many of the same scoring components and are similar in their weighting of each component. Because they are correlated, this suggests the validity of using the R2CHADS2 score to predict the risk of stroke. However, the addition of renal failure may not change clinical decisions or outcomes since the CHA2DS2VASc has already been validated and is currently being used.

These findings complement prior research highlighting a correlation between renal failure and stroke risk in patients with NVAF [13]. It also supports research suggesting the potential benefit of adding renal failure to current stroke risk prediction schemes [14]. While the risks and benefits of OAC use should always be reviewed on an individual basis, the results of this study support anticoagulation in CKD patients with NVAF in order to prevent future thromboembolic events [15].

Due to the high morbidity and mortality associated with thromboembolism, it is important to attempt to control modifiable risk factors, such as renal function. While many of the risk factors for $\mathrm{CKD}, \mathrm{AF}$, and stroke are the same, renal failure may prove to be a separate independent risk factor for thromboembolism. It would be reasonable to consider the addition of renal failure to stroke risk prediction scores to better assess the risks and benefits of OAC in patients with kidney disease.

There are several limitations to this study. First, the generalizability of the results of this study is limited, as this was a small sample collected from a single Midwest hospital over a limited two-year period. Despite the small sample size, it provides a basis for further research in evaluating the role of renal function in patients with NVAF and risk of stroke. Secondly, this study compares eGFR to both the CHA2DS2VASc and R2CHADS2 scores. These scoring schemes vary in the number of points assigned for each risk factor. Most notably, scoring for age varies widely between these two schemes. Because of this difference, it is difficult to compare these head to head. While one could argue the need for a comparable R2CHA2DS2VASc, the decision to use the R2CHADS2 score derives from its validation in predicting stroke risk [16]. 
Another limitation was the decision to use the best creatinine value during hospitalization. While this was used as the best estimate of the patient's normal baseline creatinine, it may not accurately reflect kidney function as other confounding variables may have adversely affected it during hospitalization. Thus, eGFR calculations may have been altered, although the decision to use the GFR-CKD/EPI equation over other estimated eGFR calculations, such as the Modification of Diet in Renal Disease (MDRD) equation, should improve accuracy, specifically with higher renal function [17]. A larger sample size may help to eliminate some of the potential variability in creatinine values and better aid in accurately evaluating the correlation between renal failure and stroke risk.

\section{Conclusions}

In patients with NVAF presenting with stroke, renal failure is associated with higher CHA2DS2VASc and R2CHADS2 scores. One must consider renal failure (end-stage or non-end stage renal failure) as an additional potential risk factor for stroke when recommending anticoagulation in non-valvular atrial fibrillation. There is a further need for a randomized control trial to consider renal failure as an independent risk factor for stroke in non-valvular atrial fibrillation.

\section{Additional Information}

\section{Disclosures}

Human subjects: Consent was obtained by all participants in this study. Animal subjects: All authors have confirmed that this study did not involve animal subjects or tissue. Conflicts of interest: In compliance with the ICMJE uniform disclosure form, all authors declare the following: Payment/services info: All authors have declared that no financial support was received from any organization for the submitted work. Financial relationships: All authors have declared that they have no financial relationships at present or within the previous three years with any organizations that might have an interest in the submitted work. Other relationships: All authors have declared that there are no other relationships or activities that could appear to have influenced the submitted work.

\section{References}

1. Andrade J, Khairy P, Dobrev D, and Nattel S: The clinical profile and pathophysiology of atrial fibrillation: relationships among clinical features, epidemiology, and mechanisms. Circ Res. 2014, 114:1453-1468. 10.1161/circresaha.114.303211

2. Go AS, Fang MC, Udaltsova N, et al.: Impact of proteinuria and glomerular filtration rate on risk of thromboembolism in atrial fibrillation: the anticoagulation and risk factors in atrial fibrillation (ATRIA) study. Circulation. 2009, 119:1363-1369.

10.1161/circulationaha.108.816082

3. Kim MH, Johnston SS, Chu BC, Dalal MR, Schulman KL: Estimation of total incremental health care costs in patients with atrial fibrillation in the United States. Circ Cardiovasc Qual Outcomes. 2011, 4:313-320. 10.1161/circoutcomes.110.958165

4. Fuster V, Ryden LE, Cannom DS, et al.: 2011 ACCF/AHA/HRS focused updates incorporated into the ACC/AHA/ESC 2006 Guidelines for the management of patients with atrial fibrillation: a report of the American College of Cardiology Foundation/American Heart Association Task Force on Practice Guidelines developed in partnership with the European Society of Cardiology and in collaboration with the European Heart Rhythm Association and the Heart Rhythm Society. Cardiol. 2011, 57:101-198. 10.1016/j.jacc.2010.09.013

5. Chao TF, Tsao HM, Ambrose K, at al.: Renal dysfunction and the risk of thromboembolic events in patients with atrial fibrillation after catheter ablation--the potential role beyond the CHA(2)DS(2)-VASc score. Heart Rhythm. 2012, 9:1755-1760. 10.1016/j.hrthm.2012.06.039

6. Coresh J, Selvin E, Stevens LA, et al.: Prevalence of chronic kidney disease in the United States. Jama. 2007, 298:2038-2047. 10.1001/jama.298.17.2038 
7. Soliman EZ, Prineas RJ, Go AS, et al.: Chronic kidney disease and prevalent atrial fibrillation: the Chronic Renal Insufficiency Cohort (CRIC). Am Heart J. 2010, 159:1102-110. 10.1016/j.ahj.2010.03.027

8. Masson P, Webster AC, Hong M, Turner R, Lindley RI, and Craig JC: Chronic kidney disease and the risk of stroke: a systematic review and meta-analysis. Nephrol Dial Transplant. 2015, 30:1162-1169. 10.1093/ndt/gfv009

9. Mozaffarian D, Benjamin EJ, Go AS, et al.: Heart disease and stroke statistics--2015 update: a report from the American Heart Association. Circulation. 2015, 131:29-322.

10.1161/cir.0000000000000152

10. Gage BF, Waterman AD, Shannon W, Boechler M, Rich MW, Radford MJ: Validation of clinical classification schemes for predicting stroke: results from the National Registry of Atrial Fibrillation. Jama. 2001, 285:2864-2870. 10.1001/jama.285.22.2864

11. Banerjee A, Fauchier L, Vourc'h P, et al.: Renal impairment and ischemic stroke risk assessment in patients with atrial fibrillation: the Loire Valley Atrial Fibrillation Project. Cardiol. 2013, 61:2079-2087. 10.1016/j.jacc.2013.02.035

12. Levey AS, Bosch JP, Lewis JB, Greene T, Rogers N, Roth D: A more accurate method to estimate glomerular filtration rate from serum creatinine: a new prediction equation. Modification of Diet in Renal Disease Study Group. Ann Intern Med. 1999, 130:461-70. 10.7326/0003-4819-130-6-199903160-00002

13. Guo Y, Wang H, Zhao X, et al.: Relation of renal dysfunction to the increased risk of stroke and death in female patients with atrial fibrillation. Cardiol. 2013, 168:1502-1508.

10.1016/j.ijcard.2012.12.099

14. Piccini JP, Stevens SR, Chang Y, et al.: Renal dysfunction as a predictor of stroke and systemic embolism in patients with nonvalvular atrial fibrillation: validation of the R(2)CHADS(2) index in the ROCKET AF (Rivaroxaban Once-daily, oral, direct factor Xa inhibition Compared with vitamin K antagonism for prevention of stroke and Embolism Trial in Atrial Fibrillation) and ATRIA (AnTicoagulation and Risk factors In Atrial fibrillation) study cohorts. Circulation. 2013, 127:224-232. 10.1161/circulationaha.112.107128

15. Bonde AN, Lip GY, Kamper, AL, et al.: Net clinical benefit of antithrombotic therapy in patients with atrial fibrillation and chronic kidney disease: a nationwide observational cohort study. Cardiol. 2014, 64:2471-2482. 10.1016/j.jacc.2014.09.051

16. Kornej J, Hindricks G, Kosiuk J, et al.: Renal dysfunction, stroke risk scores (CHADS2, CHA2DS2-VASc, and R2CHADS2), and the risk of thromboembolic events after catheter ablation of atrial fibrillation: the Leipzig Heart Center AF Ablation Registry. Circ Arrhythm Electrophysiol. 2013, 6:868-874. 10.1161/circep.113.000869

17. Levey AS, Stevens LA, Schmid CH, et al.: A new equation to estimate glomerular filtration rate. Ann Intern Med. 2009, 150:604-612. 10.7326/0003-4819-150-9-200905050-00006 